\title{
A case series analysing patients with dental anxiety: a patient-centred model based on psychological profiling
}

\section{[version 1; peer review: 1 approved with reservations, 1 not}

\section{approved]}

\author{
Riccardo Tizzoni (D1), Laura Veneroni2, Alfonso D'Aloia3, Marta Tizzoni', \\ Carlo Alfredo Clerici 4,5 \\ ${ }^{1}$ Independent Researcher, Milano, Italy \\ 2Paediatric Oncology Unit, Fondazione IRCCS Istituto Nazionale Tumori, Milano, Italy \\ ${ }^{3}$ Department of Anesthesiology, Intensive Care and Pain Therapy Dept, ASST G. Pini-CTO Hospital, Milano, Italy \\ ${ }^{4}$ Department of Oncology and Haematology-Oncology, University of Milano, Milano, Italy \\ ${ }^{5}$ Clinical Psychology Unit, Fondazione IRCCS Istituto Nazionale Tumori, Milano, Italy
}

V1 First published: 01 Nov 2019, 8:1843

https://doi.org/10.12688/f1000research.20712.1

Latest published: 04 Sep 2020, 8:1843

https://doi.org/10.12688/f1000research.20712.2

\section{Abstract}

Anxiety and distress can jeopardize dental care experience of patients and may affect the clinical result. Although a wide range of sedation and analgesia techniques are currently available to relieve distress and pain during dental procedures, operative models to choose the most effective sedation-analgesic strategies are lacking.

This case series proposes a patient-centred model to optimize patients' cooperation during dental care delivery. We describe how to achieve correct anaesthesia by using the least sedative procedure, accounting for the dental procedure needed and patient's

psychological profile.

Five patients were considered as paradigmatic to show the balance between patients' subjective experiences and the clinical procedures: a patient with low stress, good compliance (case 1); moderate stress and reduction in compliance (case 2); anxious patient (case 3); patient with acute anxiety and emotional distress (case 4); anguished patient (case 5).

A multimodal treatment of emotional and behavioural condition and a patient-centred model approach contributed to achieve the best patient satisfaction in the five cases detailed here.

\section{Keywords}

dental anxiety, sedation, analgesia, compliance, patient-centred model

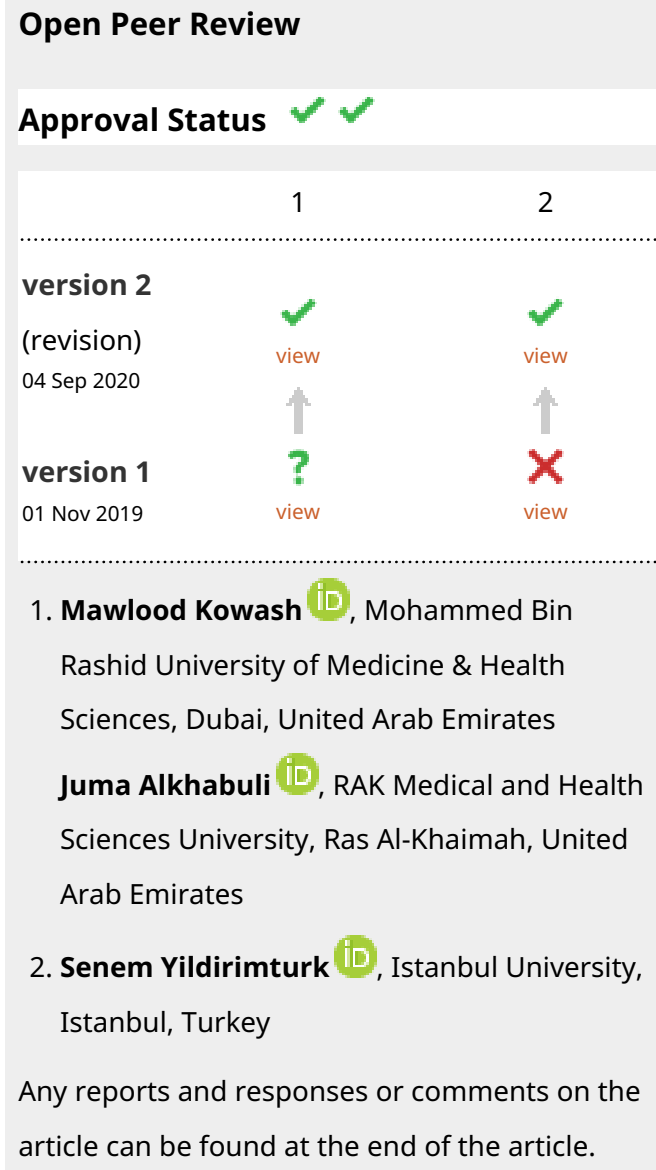

1 2

version 2

(revision)

04 Sep 2020

version 1

01 Nov 2019

$\begin{array}{cc}\checkmark \\ \text { view } \\ ? & \text { view } \\ \text { view } & \text { view }\end{array}$

1. Mawlood Kowash ID, Mohammed Bin Rashid University of Medicine \& Health Sciences, Dubai, United Arab Emirates Juma Alkhabuli ID, RAK Medical and Health Sciences University, Ras Al-Khaimah, United Arab Emirates

2. Senem Yildirimturk ID, Istanbul University, Istanbul, Turkey

Any reports and responses or comments on the article can be found at the end of the article. 
Corresponding author: Riccardo Tizzoni (dottori.tizzoni@gmail.com)

Author roles: Tizzoni R: Conceptualization, Data Curation, Investigation, Methodology, Writing - Original Draft Preparation, Writing Review \& Editing; Veneroni L: Conceptualization, Data Curation, Investigation, Writing - Review \& Editing; D'Aloia A: Conceptualization, Data Curation, Investigation, Methodology, Supervision, Writing - Review \& Editing; Tizzoni M: Conceptualization, Data Curation, Validation, Writing - Review \& Editing; Clerici CA: Conceptualization, Data Curation, Formal Analysis, Methodology, Validation, Writing Review \& Editing

Competing interests: No competing interests were disclosed.

Grant information: The author(s) declared that no grants were involved in supporting this work.

Copyright: (c) 2019 Tizzoni R et al. This is an open access article distributed under the terms of the Creative Commons Attribution License , which permits unrestricted use, distribution, and reproduction in any medium, provided the original work is properly cited.

How to cite this article: Tizzoni R, Veneroni L, D'Aloia A et al. A case series analysing patients with dental anxiety: a patient-centred model based on psychological profiling [version 1; peer review: 1 approved with reservations, 1 not approved] F1000Research 2019, 8:1843 https://doi.org/10.12688/f1000research.20712.1

First published: 01 Nov 2019, 8:1843 https://doi.org/10.12688/f1000research.20712.1 


\section{Introduction}

An increasing number of patients are undergoing day-case dental procedures or surgeries, and in some cases they may experience significant emotional upset for either consultation or dental therapy. Patients are often required to cooperate for a long time and stay in a coerced position: this may affect their psychological state and induce discomfort, fear, anxiety, and pain ${ }^{1}$. When patients can arduously cooperate during treatments, an appropriate analgesia should be achieved and a satisfactory anxiolysis should be accomplished. To this aim, a range of techniques, from the tell-show-do approach to conscious sedation and general anaesthesia, is currently available ${ }^{2,3}$.

When fearful patients cannot be reassured by explanation and comforting professional behaviour, the clinician should consider a well-tolerated and effective sedative agent, associated with topical and/or injectable anaesthetics.

The plurality of strategies available enable the team to safely provide a comprehensive care to minimize patient discomfort. Treatments include anxiolysis with oral benzodiazepines, conscious sedation with nitrous oxide, intravenous conscious sedation with benzodiazepines (with lidocaine and prilocaine cream as an aid for venepuncture), premedication for anaesthesia induction, and general anaesthesia ${ }^{4-8}$.

These techniques should be tailored based on specific individual needs and safety, to minimize anxiety, pain and memory of the dental procedure. To date in the scientific literature, there is a lack of evidence based procedures to ensure patient's compliance, considering both safety and cost-to-benefit ratio.

In this case series, five clinical cases ( 2 men and 3 women) and the procedures adopted to manage pain, behaviour and discomfort are analysed. We applied a patient-centred model to maintain patient's cooperation and manage patient's response to distress according to his/her psychological profile and the planned dental therapies.

\section{Case 1: a patient with low stress, good compliance}

A 38-year-old woman came to our private practice for longterm periodontal disease: she suffered spontaneous bleeding during the night and during dental hygiene, halitosis, and had difficulty in hygiene maintenance. At the first visit, the patient was relaxed and confident with the environment and professionals. After a comprehensive periodontal visit, the patient received all the information on her disease and house hygiene maintenance and two dental chair appointments for dental hygiene were scheduled. We discussed the available procedures for pain and distress control; however, she refused any medication as she exhibited very low stress and good compliance. She only accepted pre-anaesthetic liquid on the gums (Lidocaine 15\% spray). Both cleaning procedures were performed without distress and the patient was successfully treated for an initial form of periodontitis. Afterwards, the patient was included in a six month-recall programme for dental hygiene. The patient reported in each follow-up visit satisfaction for the previous treatment.
For patients with low stress and good compliance and undergoing dental treatments or procedures, such as dental hygiene, X-ray examinations, impression taking, simple stages of prosthetic rehabilitation and restorative dentistry, dentist behaviour and the use of pre-anaesthetic paste or liquid are enough to control pain and manage the patient.

\section{Case 2: a patient with moderate stress and reduced compliance}

A 34-year-old woman was referred to our private practice by a psychologist: she had suffered panic attacks and agoraphobia and had been in psychological therapy for about five years. The patient needed professional dental hygiene and some fillings.

At the first visit, the patient revealed slightly uneasiness and was unsettled and tense: she disclosed her discomfort while being subjected to dental procedures and enduring long dental chair appointments and she was worried about potential pain. In agreement with her psychologist, we decided to supply her with local analgesia (Pre-anaesthetic liquid: Lidocaine $15 \%$ spray; injected local anaesthesia: Mepicain 2\%, $1.8 \mathrm{ml}$, 1:100.000 adrenaline, or Mepicain 3\%, $1.8 \mathrm{ml}$, without adrenaline) and benzodiazepine per os (10 drops of bromazepam were administered, 30 minutes before each dental procedure). Dental procedures were performed within a reasonable length of time with the patient remaining comfortable throughout the dental chair appointments. The patient reported to her psychologist satisfaction for the received treatments and, thus, the same regimen of anxiolysis was maintained during the following dental chair appointments.

Restorative dentistry, endodontic therapies, scaling and rootplanning therapies are generally well tolerated since they have a low intensity of physical discomfort and low grade of psychological effort. However, for certain patients, the use of per os benzodiazepine is advisable in addition to local anaesthesia.

\section{Case 3: an anxious patient}

A 54-year-old male needed the extraction of an upper wisdom tooth; the patient complained of pain, posteriorly, to the upper left side of the maxilla. After X-ray examination, the upper left wisdom tooth was diagnosed with a carious lesion with pulpal involvement, causing the pain.

The patient seemed to be cooperative, compliant and confident with the professionals and the surgery, even though the clinical history and medical record showed previous episodes of moderate anxiety related to dental procedures. Before surgical intervention, a pre-anaesthetic liquid (Lidocaine 15\% spray) was used and local injected anaesthesia were administered on the vestibular and palatal aspects, for both pain control and vasoconstriction (Ecocain, 2x $20 \mathrm{mg} / \mathrm{ml}, 1,8 \mathrm{ml}$, 1:50:000 adrenaline). However, due to pulpal involvement and the presence of an infection, good pain control was not achievable ${ }^{9}$ : immediately at the beginning of the procedure, the use of periosteals to break and cut gingival fibres and the use of forceps to move the tooth for dislocation caused intense 
pain. The patient showed clear signs and symptoms of distress and an inability to withstand that surgical situation. Thus, he rapidly became nervous and agitated. To not lose the confidence and trust of the patient, we administered benzodiazepine (bromazepam 15 drops per os) and nitrous oxide (Inhalation; Start: $10 \%$ nitrous oxide and $90 \%$ oxygen, progressively reaching $40 \%$ nitrous oxide and $60 \%$ oxygen). After 20 minutes of relaxation, we could perform the extraction with the perfect compliance of the patient and better control of intraoperative pain. At the end of the procedure, we administered $100 \%$ oxygen for 10 minutes for patient recovery. The patient reported satisfaction for previous treatment at the suture removal visit.

When facing a patient with special needs (anxiety and reduced compliance), especially before procedures such as a planned minor surgery, or an extraction or a mucogingival surgery or an osseous periodontal surgery or a simple case of implantology, the dental team should preserve patient confidence, reduce anxiety and obtain compliance. In addition to local anaesthesia, the dentist should be able to manage benzodiazepine per os associated with nitrous oxide administration, even in absence of the specialist anaesthesiologist.

The association of benzodiazepine and nitrous oxide positively affects both the patient and the dental professional: in fact, a good disturbance of time and space perception and deep conscious sedation are achievable by adjusting nitrous oxide titration; the dentist is more relaxed and concentrated on the procedure. Analgesia is then well controlled by means of local anaesthesia.

\section{Case 4: a patient with acute anxiety and emotional distress}

A 58-year-old female in good general health status required some restorative dentistry and a complex implant-prosthetic rehabilitation on both arches.

She suffered from acute anxiety and emotional distress; she constantly asked for explanations before and after each planned dental treatment, with an extreme waste of time during dental chair appointments. The dental treatment presupposed sinus lift procedures on both sides of the maxilla and concomitant implant placement. Bone harvesting from the malar surface of both maxillary bones by a scraper was planned. Therefore, the planned procedure was complex and poorly accepted by patient. For this reason and accounting for her emotional status, the patient underwent intravenous sedation (Diazepam: starting dose $7 \mathrm{mg}$; then $0.6 \mathrm{mg}$ every 25 minutes were administered up to 20 minutes before the end of the procedure). A 2-hour observation period followed the surgery to allow full recovery of the patient. Administering sedation and postoperatively anti-inflammatory drugs intravenously was helpful. A good disturbance of time and space perception was obtained, with complete recovery and full patient's satisfaction. The patient maintained a good compliance and report satisfaction during the next dental chair appointment. In following appointments, for prosthetic rehabilitation completion, the patient was managed by means of per os benzodiazepines (bromazepam 15 drops 30 minutes before each dental procedure) and local anaesthesia when requested by the procedure (preanaesthetic liquid: Lidocaine $15 \%$ spray; injected local anaesthesia: Mepicain 2\%, $1.8 \mathrm{ml}, 1: 100.000$ adrenaline).

Intravenous sedation is an effective therapy for patients suffering acute anxiety and distress during dental procedures. It offers several advantages: the patient may become completely relaxed, depending on the deepness of sedation obtained by drug titration, compliant and with a good disturbance of time and space perception. In this condition, even major surgery can be accomplished, with a reduced duration of dental procedure and lower stress for the professional. Intravenous sedation has been successfully utilised for complex surgical clinical cases, such as bilateral sinus lifting and bone grafts and implants or bilateral vertical and lateral augmentation procedures with bone harvesting. Through the same vein utilised to administer conscious sedation drugs, it is advisable to administer also anti-inflammatory drugs in the immediate postoperative phase. The patient should be in good health and it is desirable to give the patient postoperative time for recovery and to have someone accompanying him/her back home. The dental office should have a good professional relationship with a specialist anaesthesiologist and higher costs should be expected for the patient.

\section{Case 5: an anguished patient}

A 50-year-old male in excellent general health condition needed to undergo fixed implant-supported dental rehabilitation. Despite the strong motivation to be rehabilitated and the awareness of the related surgeries, at the first visit the patient was stressed about dental therapies, exhibiting distress and anguish.

From an oral point of view, the patient was afflicted by severe periodontal disease. He lacked all the teeth of the upper left hemi-maxilla, except the central incisor; bone reabsorption of the area was massive with the tongue interposing between the arches laterally. The periodontal disease was progressively well controlled by proper therapies and a bone grafting at the left side of the maxilla was planned. According to the literature, autogenous bone is considered as the gold standard for bone grafting procedures ${ }^{10}$; therefore, calvaria was selected as the donor site, to harvest bone blocks enough to perform vertical and horizontal bone augmentation in a large area for proper dental implants insertion. Sinus lifting was planned concomitantly with bone augmentation. In this case, the unique appropriate sedation technique was general anaesthesia (Propofol; Starting dose: $144 \mathrm{mg}$; then $640 \mathrm{mg}$ per hour of continuous venous infusion up to 15 minutes before the end of the surgical procedure; then $320 \mathrm{mg}$ per hour of continuous venous infusion up to 5 minutes before the end of the surgical procedure; then the infusion was stopped). Local anaesthesia was also administered in the mouth and in the parietal bone area of the patient, for both pain control and vasoconstriction (Ecocain, $6 \times 20 \mathrm{mg} / \mathrm{ml}, 1.8 \mathrm{ml}, 1: 50: 000$ adrenaline). After 5 months, five endo-osseous dental implants were placed. In previous dental care procedures, the dental team gradually 
gained patient's confidence, so that the surgical stage of implant placement and other prosthetic phases were performed with local anaesthesia (Pre-anaesthetic liquid: Lidocaine 15\% spray; injected local anaesthesia: Mepicain 2\%, $1.8 \mathrm{~mm}, 1: 100.000$ adrenaline) or, rarely, with benzodiazepine per os (bromazepam 15 drops, 30 minutes before each treatment). All the implants were rehabilitated four months after implant placement.

General anaesthesia is of paramount importance for the anguished patient while undergoing dental treatment. It may also represent the only chance to treat children under 4 years of age or to accomplish particular surgical procedures, such as bone harvesting from hip or calvaria for successive implant stabilisation or interventions in the extreme proximity to vascular or nervous anatomical structures.

The patient should be in good general health, hospitalisation is essential for the patient as well as the presence of a specialist anaesthesiologist. Generally, it is advisable to guarantee at least 24 hours for full recovery. Costs may eventually increase.

\section{Discussion}

This case series describes five scenarios that frequently occur in clinical practice, with the evident limit of showing only few of the main typology of distress and pain management during dental procedures. Facing a wide range of patients, from relaxed and collaborative patients to anguished ones, the dental team should optimize and tailor its approach, considering both patients' psychologic profile and the planned procedures.

The applicability and, at the same time, the limit of the described approach is that the evaluation and treatment of anxiety and distress had been made by clinical observation and counselling and patient self-report, but was not based upon a mental health specialist evaluation and treatment (questionnaire of rating scale, psychotherapy or psychiatric consultation), since they are not available in a routine clinical dental practice.
During treatments, patients are required to cooperate to achieve a degree of relaxation enough to maintain the fixed positioning necessary for the treatment. Therefore, in patients with a pre-existing psychiatric history, psychosocial maladjustment or psychological problems such as anxiety and previous traumatic experiences related to dental therapies, behavioural manifestations can hinder the correct execution of procedure, safety and clinical outcomes ${ }^{11}$.

Before choosing the type of sedative-analgesic approach, the preoperative diagnosis, patient psychological profile, and the planned dental procedure should be carefully evaluated. The final aim is to perform the therapies with relaxed and cooperating patients and in an uneventful and smooth induction of anaesthesia and sedation, if needed. If well-balanced, sedation can convert the behaviour of a patient uncooperative to undergo dental treatment.

Our experience indicates that it is necessary to adopt the most appropriate combination of resources (Table 1), according to patient's characteristics and needs. Therefore, we proposed a decision-making strategy for the dental clinician, as a tool to maintain an acceptable level of collaboration and, therefore, of patient comfort all the time. Clinical management strategy, analgesic agent and sedation therapy should be driven by the subjective perception of the patient. Besides generally utilised medication for the control of the pain, the dental clinician can discuss with the patient to determine the best approach to reduce distress and anxiety and properly manage the clinical scenario, considering all available techniques of sedation.

Therefore, in this patient-centred model the clinical decision of the behavioural support, analgesia and/or sedation procedures is guided by patient complexity (Table 2). For example, a patient with strong anxiety, but with a very simple procedure may still require highly complex anxiolytic and analgesic procedures (as in cases 3 and 4). If a level is not feasible (e.g. local anaesthesia does not work for an infection), the next level of anxiolysis should be considered.

Table 1. Basic resources for the patient-centred model.

\begin{tabular}{|l|l|}
\hline Resource & The patient should be reassured and entertained before and during the dental procedure intended to \\
\hline Behaviour & $\begin{array}{l}\text { The accomplished. Explanations must be given before each treatment. } \\
\text { be }\end{array}$ \\
\hline $\begin{array}{l}\text { Local } \\
\text { anaesthesia }\end{array}$ & $\begin{array}{l}\text { Topical and/or injectable anaesthetics and, if needed, sedatives can be chosen to both achieve pain } \\
\text { relief and gain patient's compliance and cooperation. Failure to achieve complete local anaesthesia is } \\
\text { not uncommon in the management of orofacial infections }{ }^{9} \text { : a shift to a deeper sedation may be required. }\end{array}$ \\
\hline Anxiolytics & $\begin{array}{l}\text { Use of anxiolytic drugs may be indicated to manage some disorders that cannot be addressed only } \\
\text { with psychological and relational tools but need psychopharmacological therapy; the use of low-dose } \\
\text { benzodiazepines to reduce anxiety can be very helpful. Patients' responses to medications and doses } \\
\text { can vary dramatically (i.e. from a moderate sedation to unintended deep sedation). }\end{array}$ \\
\hline $\begin{array}{l}\text { Procedural } \\
\text { sedation }\end{array}$ & $\begin{array}{l}\text { A complex of techniques to manage patient's pain and anxiety in a safe, effective and human fashion } \\
\text { by maintaining spontaneous breathing and airway-protective reflexes }{ }^{8} \text {. The patient should be carefully } \\
\text { evaluated and prepared before sedation and monitored during the procedure and recovery. Sedation } \\
\text { could be titrated and reduced in future treatments, based on the patient's response. This technique is } \\
\text { specially indicated for those individuals who are terrified of dental treatment. }\end{array}$ \\
\hline $\begin{array}{l}\text { General } \\
\text { anaesthesia }\end{array}$ & $\begin{array}{l}\text { It requires an accurate evaluation of costs and benefits, the presence of good general conditions, } \\
\text { hospitalisation, and the presence of an anaesthesiologist. }\end{array}$ \\
\hline
\end{tabular}


Table 2. Modulating and balancing behaviour and/or analgesia and/or sedation in response to an increasing complexity and/or length of the procedure and increasing level of anxiety and distress. As an example, when it is not possible to use more analgesia or sedation, it is convenient to use all behavioural techniques to perform a complex procedure with a difficult patient.

\begin{tabular}{|l|l|l|l|}
\hline Procedure & Behaviour & Analgesia & Sedation \\
\hline & + & + & + \\
\hline & ++ & + & 0 \\
\hline From degree 0 to +++ & ++ & 0 & + \\
\hline & + & ++ & 0 \\
\hline & + & ++ & + \\
\hline & 0 & 0 & ++ \\
\hline & +++ & 0 & 0 \\
\hline 0 & + & ++ \\
\hline & 0 & 0 & 0 \\
\hline
\end{tabular}

0 : no increment of behaviour, analgesia or sedation; + : small increment of behaviour, analgesia or sedation; ++ : medium increment of behaviour, analgesia or sedation; +++: high increment of behaviour, analgesia or sedation.
Along specific goals of sedation, the safety and prompt recovery to a state of consciousness must be considered. Furthermore, dentist's preparation, expertise and experience can decrease the duration of the procedure, thereby limiting the need for sedation and analgesia ${ }^{12,13}$.

Nevertheless, from our experience we learnt that it is still a subjective process and each clinician must always be aware of how the patient is responding to sedatives. The more complex and/or as the length of the procedure increases, the more the dentist's behaviour and/or analgesia and/or sedation need to match the new situation. A patient-centred approach still needs more studies to validate operative models to choose sedation and analgesic strategies to achieve the best patient satisfaction.

In conclusion, a patient-centred approach considering both clinical characteristics and psychological profile can help to achieve a tailored management of pain control and sedation in patients with emotional and behavioural problems during dental procedures.

\section{Consent}

All patients gave their written informed consent to publish the data presented in this case series.

\section{Data availability}

All data underlying the results are available as part of the article and no additional source data are required.
1. Facco E, Zanette G: The Odyssey of Dental Anxiety: From Prehistory to the Present. A Narrative Review. Front Psychol. 2017; 8: 1155. PubMed Abstract | Publisher Full Text | Free Full Text

2. Carter $\mathrm{AE}$, Carter $\mathrm{G}$, Boschen $\mathrm{M}$, et al:: Pathways of fear and anxiety in dentistry: A review. World J Clin Cases. 2014; 2(11): 642-53. PubMed Abstract | Publisher Full Text | Free Full Text

3. Araújo JO, Motta RHL, Bergamaschi CC, et al.: Effectiveness and safety of oral sedation in adult patients undergoing dental procedures: protocol for a systematic review. BMJ Open. 2018; 8(1): e017681. PubMed Abstract | Publisher Full Text | Free Full Text

4. Sebastiani FR, Dym H, Wolf J: Oral Sedation in the Dental Office. Dent Clin North Am. 2016; 60(2): 295-307.

PubMed Abstract | Publisher Full Text

5. Armfield JM, Heaton LJ: Management of fear and anxiety in the dental clinic: a review. Aust Dent J. 2013; 58(4): 390-407. PubMed Abstract | Publisher Full Text

6. O'Halloran M: The use of anaesthetic agents to provide anxiolysis and sedation in dentistry and oral surgery. Australas Med J. 2013; 6(12): 713-8. PubMed Abstract | Publisher Full Text | Free Full Text

7. Brown TB, Lovato LM, Parker D: Procedural sedation in the acute care setting. Am Fam Physician. 2005; 71(1): 85-90. PubMed Abstract

8. Appukuttan DP: Strategies to manage patients with dental anxiety and dental phobia: literature review. Clin Cosmet Investig Dent. 2016; 8: 35-50. PubMed Abstract | Publisher Full Text | Free Full Text

9. Fleury AA: Local anesthesia failure in endodontic therapy: the acute inflammation factor. Compendium. 1990; 11(4): 210, 212, 214 passim. PubMed Abstract

10. Shamsoddin E, Houshmand B, Golabgiran M: Biomaterial selection for bone augmentation in implant dentistry: A systematic review. $J$ Adv Pharm Technol Res. 2019; 10(2): 46-50.

PubMed Abstract | Publisher Full Text | Free Full Text

11. De Jongh A, Adair $P$, Meijerink-Anderson $M$ : Clinical management of dental anxiety: what works for whom? Int Dent J. 2005; 55(2): 73-80. PubMed Abstract | Publisher Full Text

12. Yamalik N: Dentist-patient relationship and quality care 3. Int Dent J. 2005; 55(4): 254-6.

PubMed Abstract | Publisher Full Text

13. American Society of Anesthesiologists Committee on Standards and Practice Paramet: Practice Guidelines for Moderate Procedural Sedation and Analgesia 2018: A Report by the American Society of Anesthesiologists Task Force on Moderate Procedural Sedation and Analgesia, the American Association of Oral and Maxillofacial Surgeons, American College of Radiology, American Dental Association, American Society of Dentist Anesthesiologists, and Society of Interventional Radiology. Anesthesiology. 2018; 128(3): 437-79. PubMed Abstract | Publisher Full Text 


\section{Open Peer Review}

\section{Current Peer Review Status: ? $\mathrm{X}$}

\section{Version 1}

Reviewer Report 13 July 2020

https://doi.org/10.5256/f1000research.22779.r65973

(C) 2020 Yildirimturk S. This is an open access peer review report distributed under the terms of the Creative Commons Attribution License, which permits unrestricted use, distribution, and reproduction in any medium, provided the original work is properly cited.

\section{Senem Yildirimturk}

Department of Oral and Maxillofacial Surgery, Istanbul University, Istanbul, Turkey

Here are my comments to the authors of 'A case series analysing patients with dental anxiety: a patient-centred model based on psychological profiling [version 1; peer review: 1 approved with reservations]':

1. If this study is an original research/research article, then what is the null hypothesis of the study? If it is a review, the authors should better enhance the discussion and strongly support it with literature conducting dental anxiety, MDAS, sedation, etc. The examples given in the study (one in each type) are not enough to make a review!

2. The introduction and discussion parts are too short.

3. The authors should better use valid scales or questionnaires to avoid subjectivity. The dental history of the patient mostly affects the current state/mood on the day or even before the day of dental appointment regardless of operation type.

4. The grammar is poor.

5. The aim and methodology of the study should be revised and planned in detail.

Is the background of the cases' history and progression described in sufficient detail? No

Are enough details provided of any physical examination and diagnostic tests, treatment given and outcomes?

No

Is sufficient discussion included of the importance of the findings and their relevance to future understanding of disease processes, diagnosis or treatment? 
No

Is the conclusion balanced and justified on the basis of the findings?

Partly

Competing Interests: No competing interests were disclosed.

Reviewer Expertise: Oral surgery, sedation, dental fear, dental anxiety, oral radiology, cbct, dental implantology, facial fractures, oral and maxillofacial surgery

I confirm that I have read this submission and believe that I have an appropriate level of expertise to state that I do not consider it to be of an acceptable scientific standard, for reasons outlined above.

Reviewer Report 07 July 2020

https://doi.org/10.5256/f1000research.22779.r65974

(C) $\mathbf{2 0 2 0}$ Kowash $\mathbf{M}$ et al. This is an open access peer review report distributed under the terms of the Creative Commons Attribution License, which permits unrestricted use, distribution, and reproduction in any medium, provided the original work is properly cited.

\section{Mawlood Kowash}

Hamdan Bin Mohammed College of Dental Medicine, Mohammed Bin Rashid University of Medicine \& Health Sciences, Dubai, United Arab Emirates

Juma Alkhabuli

RAK Medical and Health Sciences University, Ras Al-Khaimah, United Arab Emirates

The five-case series of adult patients with varying degrees of anxiety and dental treatment proposes a patient-centred model to optimize patients' cooperation during dental care delivery. Dental treatment of the five patients was achieved with behaviour management and local analgesia alone or with oral and inhalation conscious sedation, intravenous sedation, or general anaesthesia.

The reviewers suggest the following corrections to make the paper scientifically sound: English language revision, revising the introduction by adding: 1) a paragraph on definition and assessment of dental anxiety; 2) a paragraph on conscious sedation, patient's assessment, and monitoring. The discussion may be improved by revising Table 2 and adding a paragraph to emphasize the importance of communication and utilizing the different behaviour management techniques to manage patient dental anxiety. Finally, revision of the conclusion is suggested.

English Language Revision: the whole paper needs English language editing. The authors should use the commonly used words/terms in academic writing.

In the introduction: $1^{\text {st }}$ paragraph: change the word "for" to "from" either consultation or dental therapy. $3^{\text {rd }}$ paragraph: use of topical analgesia prior to Local analgesia (LA) injection- and not "venipuncture" which means intra-venous access. 
In Case 1, line 7: "House hygiene maintenance" to be changed in to "oral hygiene maintenance".

The authors used the term (analgesia) in the abstract which is the correct term for dental local analgesia (LA). However, they also used (anaesthesia) throughout the paper to describe dental LA. Analgesia means the absence of pain sensation, while anesthesia means loss of all sensation including pressure and proprioception. Therefore, we suggest changing the term "anaesthesia" with 'analgesia" for dental LA.

The abbreviations should be defined when first used in the paper: e.g. Benzodiazepine per os: is OS mean by oral route?

Use the word "restorations" instead of "fillings"

In case 2: First paragraph: define "agoraphobia": extreme or irrational fear of entering open or crowded places, of leaving one's own home, or of being in places from which escape is difficult.

In $2^{\text {nd }}$ paragraph, line 5 "we decided to supply her...should be changed to "The local analgesia is applied

Case 3: In the last paragraph: change the word "association" to "combination" of benzodiazepine and nitrous oxide positively affects ....

Case 5: line 8: change "reabsorption" to "resorption".

\section{Revision of Cases}

Case 1

Start with the patient complaint or the reason for attending (e.g. refereed by..) and not by diagnosis.

It is not clear how the stress level was evaluated or based on which criteria?

\section{Case 2}

- Consultation with the patient's psychologist should have been done prior to subjecting the patient to complex dental treatment.

The following statement needs revision because the mentioned dental procedures are anxiety and pain-provoking procedures and generally are not tolerated by anxious patients: "Restorative dentistry, endodontic therapies, scaling and root planning therapies are generally well tolerated since they have a low intensity of physical discomfort and low grade of psychological effort. However, for certain patients, the use of per os benzodiazepine is advisable in addition to local anaesthesia."

\section{Case 3}

Needs language revision as shown:

A 54-year-old male needed the extraction of an upper wisdom tooth; the patient complained of pain posteriorly, to (on) the upper left side of the maxilla. After X-ray clinical and radiographic examinations, the upper left wisdom tooth was found diagnosed with deeply a carious lesion with pulpal involvement, causing the pain.

As mentioned in case 2, for the patient presented in Case 3 proper treatment planning should have been carried out because "clinical history and medical record showed previous episodes of moderate anxiety related to dental procedures". In addition, he required extraction of pulpally involved upper third molar. The infiltration LA should have been 
supplemented with inhalation sedation and/or benzocaine oral sedation.

In the second paragraph, line 11: following sentence needs revision:

"immediately at the beginning of the procedure, the use of periosteals to break and cut gingival fibres and the use of forceps to move luxate the tooth for dislocation caused intense"

\section{Case 4}

The following statement is inappropriate:

"She suffered from acute anxiety and emotional distress; she constantly asked for explanations before and after each planned dental treatment, with an extreme waste of time during dental chair appointments".

The procedures should have been explained to the patient in simple language with empathy, taking into consideration her acute anxiety and emotional stress. Treatment under IV sedation should have been planned because she was diagnosed with acute anxiety and emotional stress.

A good "disturbance" to be changed to "consciousness" of time and space perception..."

Introduction- Add the following:

\section{Definition and assessment of dental anxiety}

The term Dental Anxiety (DA) includes anxiety, fear and phobia which are used interchangeably (McDonnell-Boudra et al. 2014). DA is a reaction to unknown perceived dental danger especially when the treatment proposed was never experienced before. Dental fear is a reaction to a known perceived danger which involves a flight-or-fight response when provoked with the frightening stimulus, while dental phobia is an extreme, marked, and persistent fear of clearly visible defined objects or situations.

Understanding the level of patient anxiety allows its appropriate management. However, anxiety is difficult to measure. There are several methods available for dentist to score patients dental anxiety for example the Modified Child Dental Anxiety Scale (MCDAS) (Wong et al. 1998).

\section{Definition of conscious sedation, patient's assessment, and monitoring}

Conscious sedation is a drug-induced depression of consciousness where the patient purposefully responds to verbal commands, either alone or by light tactile stimulation. No interventions are required to maintain a patent airway, and spontaneous ventilation is adequate. Patient's cardiovascular function is usually maintained (Galeotti et al. 2016).

Meticulous pre-sedation evaluation with respect to patient's general health status, airway, fasting, and understanding about the pharmacodynamics and pharmacokinetics of the drugs must be recognized. Availability of airway management equipment, sedative drugs' antidote, venous access, suitable intraoperative monitoring such as pulse oximetry and well-trained staff must be ensured (Attri et al. 2017). Conscious sedation can be administered through various routes such as oral, intramuscular, intravenous, and inhalational.

McDonnell-Boudra D, Martin A, Hussein I. In vivo exposure therapy for the treatment of an adult needle phobic. Dent Update. 2014;41(6):533-40.

Wong HM, Humphris GM, Lee GTR. Preliminary validation and reliability of the modifed child dental anxiety scale. Psychol Rep. 1998; 83:1179-86. 
Galeotti A, Garret Bernardin A, D'Antò V, Ferrazzano GF, Gentile T, Viarani V, et al. Inhalation conscious sedation with nitrous oxide and oxygen as alternative to general anesthesia in precooperative, fearful, and disabled pediatric dental patients: A large survey on 688 working sessions. Biomed Res Int 2016. 2016:7289310.

Attri JP, Sharan R, Makkar V, Gupta KK, Khetarpal R, Kataria AP, et al. Conscious sedation: Emerging trends in pediatric dentistry. Anesth Essays Res. 2017; 11:277-81.

\section{Discussion}

In table 2 the highlighted sentence needs deletion from the title.

Table 2. Modulating and balancing behaviour and/or analgesia and/or sedation in response to an increasing complexity and/or length of the procedure and increasing level of anxiety and distress. As an example, when it is not possible to use more analgesia or sedation, it is convenient to use all behavioural techniques to perform a complex procedure with a difficult patient.

In Table 2: it is not possible to have a profound analgesia (+++) in a patient with $(0$ increment) behaviour and ( 0 increment) sedation.

Add the following at the end of the discussion:

Most anxious patients can be managed by proper communication and utilizing different behaviour management techniques (e.g. Tell-Show-Do, Distraction, Modelling, etc.) tailored to the patient psychological status. The clinician should strive to perform dental treatment using proper communication, behaviour management and local analgesia with or without oral and inhalational conscious sedation.Intravenous sedation and general anaestheisa should be reserved to complex cases to avoid potential morbidities and mortalities and additional treatment costs.

Conclusion: May be improved as follows:

In conclusion, a patient-centred approach considering both clinical characteristics and psychological profile can help to achieve high quality dental care through a tailored management of pain and anxiety in patients with emotional and behavioural problems. This can be achieved by proper behavior management and local analgesia with or without conscious sedation or in complex cases using intravenous sedation or general anaesthesia.

Is the background of the cases' history and progression described in sufficient detail? Partly

Are enough details provided of any physical examination and diagnostic tests, treatment given and outcomes?

Partly

Is sufficient discussion included of the importance of the findings and their relevance to future understanding of disease processes, diagnosis or treatment?

Partly

Is the conclusion balanced and justified on the basis of the findings? Partly

Competing Interests: No competing interests were disclosed. 
Reviewer Expertise: Dental management of patients with special needs and behavioural problems.

We confirm that we have read this submission and believe that we have an appropriate level of expertise to confirm that it is of an acceptable scientific standard, however we have significant reservations, as outlined above.

Author Response 23 Jul 2020

Riccardo Tizzoni, Independent Researcher, Milano, Italy

Object: revisions to article A case series analysing patients with dental anxiety: a patient-centred model based on psychological profiling

Dear Reviewer,

Thank you for reviewing our manuscript. The comments and suggestions indicated by you have been precisely considered and we hope satisfactorily addressed.

Enclosed please find the revised version that incorporates the reviewers' comments as follows:

REVIEWER:

1. The language of the whole article was revised.

2. We changed the word "for" with "from".

3. We have explained the use of topical analgesia for the skin prior to venipuncture.

4. We specified the term "topical analgesia" prior to "local analgesia".

5. We changed "House hygiene maintenance" to "oral hygiene maintenance".

6. We changed the word, when appropriated, "anaesthesia" with "analgesia".

7. We specified the term "per os" (that means "by oral route") when first used in the paper.

8. We changed the world "fillings" with term "restorations" as suggested.

9. We explainded the meaning of agoraphobia (extreme or irrational fear of entering open or crowded places, of leaving one's own home, or of being in places from which escape is difficult)

10. We modified the sentence "we decided to supply her" with "the local analgesia is applied"

11. We changed the word "association" with "combination" in case 3

12. We changed the word "reabsorption" with "resorption"

\section{REVISION OF CASES}

\section{Case 1}

We started with the patient complaint or the reason for attending and not by diagnosis

The stress level was evaluated by clinical observation and self report of the patient (and we added in Discussion this period: "The applicability and, at the same time, the limit of the described approach is that the evaluation and treatment of anxiety and distress had been made by clinical observation and counselling and patient self- 
report, but was not based upon a mental health specialist evaluation and treatment (questionnaire of rating scale, psychotherapy or psychiatric consultation), since they are not available in a routine clinical dental practice)".

\section{Case 2}

The Consultation with the patient's psychologist has been done prior to subjecting the patient to complex dental treatment.

We slightly modified the meaning of the sentence as requested.

\section{Case 3}

We revised the first paragraph as indicated.

For point 2 of case 3, please see point 2 of Case 1.

For the patient presented in Case 3 also we clarified proper treatment planning with phrase "supplemental infiltration with inhalation sedation with nitrous oxide administration and / or oral sedation".

In the second paragraph, line 11 we deleted the indicated phrase, and we Case 4 emphasized patient inability to withstand the surgical situation and surgical steps.

We modified the phrase correctly indicated as inappropriate:

We changed to "consciousness" of time and space perception..."

We added:

1. a paragraph on definition and assessment of dental anxiety; 2) a paragraph on conscious sedation, patient's assessment, and monitoring.

2. We revised Table 2 and added a paragraph to emphasize the importance of communication and utilizing the different behaviour management techniques to manage patient dental anxiety.

3. We added the suggested paragraph in discussion.

4. We revised the conclusions.

In table 2 we deleted, as requested, the highlighted sentence from the title and explained the same concepts in the text.

We considered possible to add doses of local analgesia (+++) in patients in whom is not need an increment of behavioural effort and sedation.

We hope that our responses and revisions satisfy your request for revisions.

Best regards

Competing Interests: Non-Financial Competing Interests 
The benefits of publishing with F1000Research:

- Your article is published within days, with no editorial bias

- You can publish traditional articles, null/negative results, case reports, data notes and more

- The peer review process is transparent and collaborative

- Your article is indexed in PubMed after passing peer review

- Dedicated customer support at every stage

For pre-submission enquiries, contact research@f1000.com 